\title{
Anticorps monoclonaux à usage thérapeutique : spécificités du développement clinique, évaluation par les agences, suivi de la tolérance à long terme
}

\author{
Gilles Paintaud ${ }^{1}$, Marine Diviné ${ }^{2}$, Philippe Lechat ${ }^{3}$ et les participants à la table ronde $N^{\circ} 5$ de Giens XXVII* \\ 1 Université François Rabelais Tours ; CNRS UMR 7292 ; CHRU de Tours, Laboratoire de Pharmacologie-Toxicologie, Tours, France \\ 2 Amgen, France \\ 3 Afssaps, France
}

Texte reçu le 20 mars 2012 ; accepté le 4 juin 2012

\section{Mots clés :}

anticorps monoclonaux ; essais cliniques, phase I ; essais cliniques,

phase II ;

essais cliniques,

phase IV

\begin{abstract}
Résumé - Les anticorps monoclonaux (AcMo) sont très différents des autres médicaments. Notre objectif a été d'évaluer si leurs spécificités ont des conséquences sur leur développement clinique, leur évaluation par les autorités de santé et leur suivi à long terme. En ce qui concerne la relation structure-activité des AcMo, il est plus pertinent de les classer selon leur mécanisme d'action (AcMo neutralisants ou agonistes, AcMo cytolytiques) que selon leur degré d'humanisation. Des recommandations sur leur développement clinique seraient souhaitables car les phases précoces posent un certain nombre de problèmes et sont mal codifiées. La pharmacocinétique est très différente de celle des autres médicaments. L'étude de la relation concentrationeffet est difficile car les biomarqueurs sont souvent déconnectés de l'effet thérapeutique. La méthodologie de l'évaluation des AcMo par les agences et leur suivi post-autorisation de mise sur le marché (post-AMM) ne sont pas différents de ceux des autres médicaments mais les AcMo associent un ensemble de particularités.
\end{abstract}

Abréviations : voir en fin d'article.

\section{Introduction}

Les anticorps monoclonaux (AcMo) ont pris une place de plus en plus importante dans la thérapeutique et plus d'une centaine d'AcMo était en développement clinique en 2011. Ces biomédicaments (protéines recombinantes produites par des cellules en culture) ont une masse moléculaire et des mécanismes d'action très différents des médicaments "classiques », généralement obtenus par synthèse chimique. L'objectif de la table ronde était d'évaluer si les spécificités des AcMo, en termes de structure et de mode d'action, ont des conséquences sur leur développement clinique, leur évaluation par les autorités de santé et leur suivi à long terme (tolérance, bon usage et médico-économie). Seuls les AcMo « nus », c'est-à-dire non conjugués à un isotope radioactif ou à une toxine, et les immunoglobulines $\mathrm{G}(\mathrm{IgG})$ « complètes » ont été discutés, les autres formes d'AcMo constituant des cas particuliers.
Par ailleurs, les biosimilaires s'inscrivent dans un contexte très différent. Une précédente table ronde sur les AcMo, non dédiée à leur développement clinique, a servi de base aux discussions. ${ }^{[1]}$

\section{Relation structure - activité}

La plupart des AcMo thérapeutiques actuellement sur le marché sont des IgG. Ils sont composés de deux portions Fab («ab» pour antigen binding), pouvant se fixer sur l'antigène, et d'une portion $\mathrm{Fc}$ ( c » pour cristallisable), responsable des propriétés immunologiques effectrices et des propriétés pharmacocinétiques des AcMo (figure 1). Les IgG sont constituées de 2 chaînes lourdes (H) et de deux chaînes légères (L). À l'extrémité des portions Fab se trouvent les domaines variables (VH et VL), qui portent chacun 3 boucles complementarity determining region (CDR), dont

* Pour la liste des participants, voir en fin d'article. 


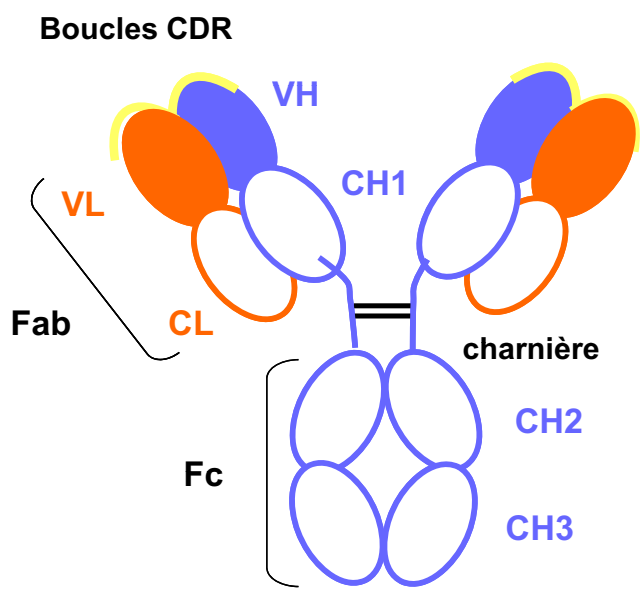

Fig. 1. Structure d'une immunoglobuline $\mathrm{G}$ (IgG). Les parties variables sont en couleur. Les portions Fab («ab» pour antigen binding), Fc («c » pour cristallisable) et les boucles CDR (complementarity determining region) sont indiquées.

l'association (6 au total) constitue le site de reconnaissance de l'épitope sur l'antigène. La portion constante (dont la séquence ne varie pas à l'intérieur d'une sous-classe d'Ig chez un individu) est composée des domaines CH1 et CL (sur la portion Fab) et des domaines $\mathrm{CH} 2$ et $\mathrm{CH} 3$ (sur la portion $\mathrm{Fc}$ ).

Les premiers AcMo étaient murins, avec pour conséquence une demi-vie courte, un faible recrutement des effecteurs immunitaires et une importante immunogénicité. Ils ont donc été progressivement humanisés, avec le développement d'AcMo chimériques (suffixe-ximab, dont seule la portion variable est murine), humanisés (suffixe -zumab, dont seuls les CDR sont murins) et enfin rendus intégralement humains (suffixe -mumab ou -umab). ${ }^{[2]}$ Cependant, du point de vue clinique, la distinction des anticorps chimériques, humanisés et totalement humains est peu pertinente car ils ont tous une portion Fc humaine et parce que certains AcMo totalement humains se sont avérés immunogènes, comme l'adalimumab (Humira $^{\circledR}$, anti-tumor necrosis factor $\alpha$ [anti$\mathrm{TNF} \alpha]$ ) dans la polyarthrite rhumatoïde (voir infra). ${ }^{[3]}$

Il est plus pertinent de classer les AcMo selon leurs mécanismes d'action. Ils peuvent en effet agir principalement soit par effet neutralisant ou agoniste, soit par effet cytolytique, en recrutant les effecteurs immunitaires (figure 2). Les anticorps neutralisants se fixent sur un antigène soluble tel qu'une cytokine, dont ils inhibent l'action, comme le fait le bevacizumab (Avastin ${ }^{\circledR}$, antivascular endothelial growth factor [anti-VEGF]) ou se fixent sur un récepteur membranaire, qu'ils bloquent, comme le font les AcMo dirigés contre la famille des récepteurs aux facteurs épidermiques (HER). Pour certains AcMo, tels que le denosumab (antireceptor activator of nuclear factor-kappaB ligand antibody [anti-RANK-ligand]), l'antigène cible existe à la fois sous forme circulante et sous forme membranaire. Cette fixation peut parfois avoir un effet agoniste si, en se fixant sur le récepteur membranaire, ils entraînent des phénomènes post-récepteur ou une apoptose, comme dans le cas des AcMo anti-TNFo. ${ }^{[4]}$ Dans le cas des effets neutralisants ou agonistes, c'est l'affinité de l'AcMo pour sa cible, donc sa portion Fab, qui est l'élément déterminant de son efficacité. Ces effets antagonistes ou agonistes ne sont pas propres aux AcMo car ils peuvent être obtenus avec des protéines de fusion ou avec des petites molécules, contrairement aux effets dépendants de la portion Fc qui sont eux spécifiques des AcMo.

Dans le cas des AcMo cytolytiques, la portion Fc joue également un rôle très important car elle est responsable du recrutement des effecteurs immunitaires : fraction $\mathrm{C} 1 \mathrm{q}$ du complément qui va entraîner une lyse cellulaire dépendante du complément (complement dependent cytotoxicity [CDC]) ou cellules effectrices porteuses du récepteur FcyRIIIA/CD16, telles que les cellules natural killer (NK) et les macrophages, qui vont entraîner une lyse dépendante des anticorps (antibody dependent cellular cytotoxicity $[\mathrm{ADCC}]) .{ }^{[5,6]} \mathrm{Il}$ existe un polymorphisme génétique se traduisant par une variation de l'affinité du récepteur FçRIIIA pour la portion $\mathrm{Fc}$ des anticorps. Il a été montré que ce polymorphisme est responsable d'une partie de la variabilité de réponse clinique aux anticorps cytolytiques tels que le rituximab (MabThera ${ }^{\circledR}$, antiCD20), ${ }^{[7]}$ le trastuzumab (Herceptin ${ }^{\circledR}$, anti-HER2) ${ }^{[8]}$ et le cetuximab (Erbitux ${ }^{\circledR}$, anti-EGFR). ${ }^{[9]}$

La portion Fc des AcMo peut par ailleurs être modifiée pour supprimer leur affinité pour les récepteurs FcyR et donc leur capacité d'ADCC et de CDC ou, au contraire, pour augmenter leur affinité et donc leurs effets cytolytiques. Ces modifications portent sur la séquence d'acides aminés ou sur la glycosylation de la portion Fc. ${ }^{[10,11]}$ Lorsque des AcMo cytolytiques sont comparés, notamment dans des modèles cellulaires, il est donc important de ne pas considérer seulement leur affinité pour l'antigène-cible mais aussi de prendre en compte les caractéristiques de leur portion Fc. Certains tests d'activité in vitro permettent d'évaluer la qualité de la portion Fc d'un AcMo quel que soit son antigène-cible. La portion Fc des AcMo influence également leur pharmacocinétique (cf. infra).

\section{Spécificité du développement clinique}

\subsection{Diversité des champs thérapeutiques et intérêt médico-économique}

En 2011, plus d'une centaine d'AcMo étaient en développement clinique. Initialement, les domaines dans lesquels les AcMo ont été développés avec succès sont la cancérologie et la rhumatologie. Depuis 1999, 29 spécialités ont obtenu une autorisation de mise sur le marché (AMM) dans 45 indications et 21 spécialités ont été examinées par la Haute autorité de santé (HAS) en vue de leur remboursement. Le premier AcMo (Orthoclone OKT3 ${ }^{\circledR}$, antiCD3), qui est murin, a été commercialisé en 1986. Le premier 


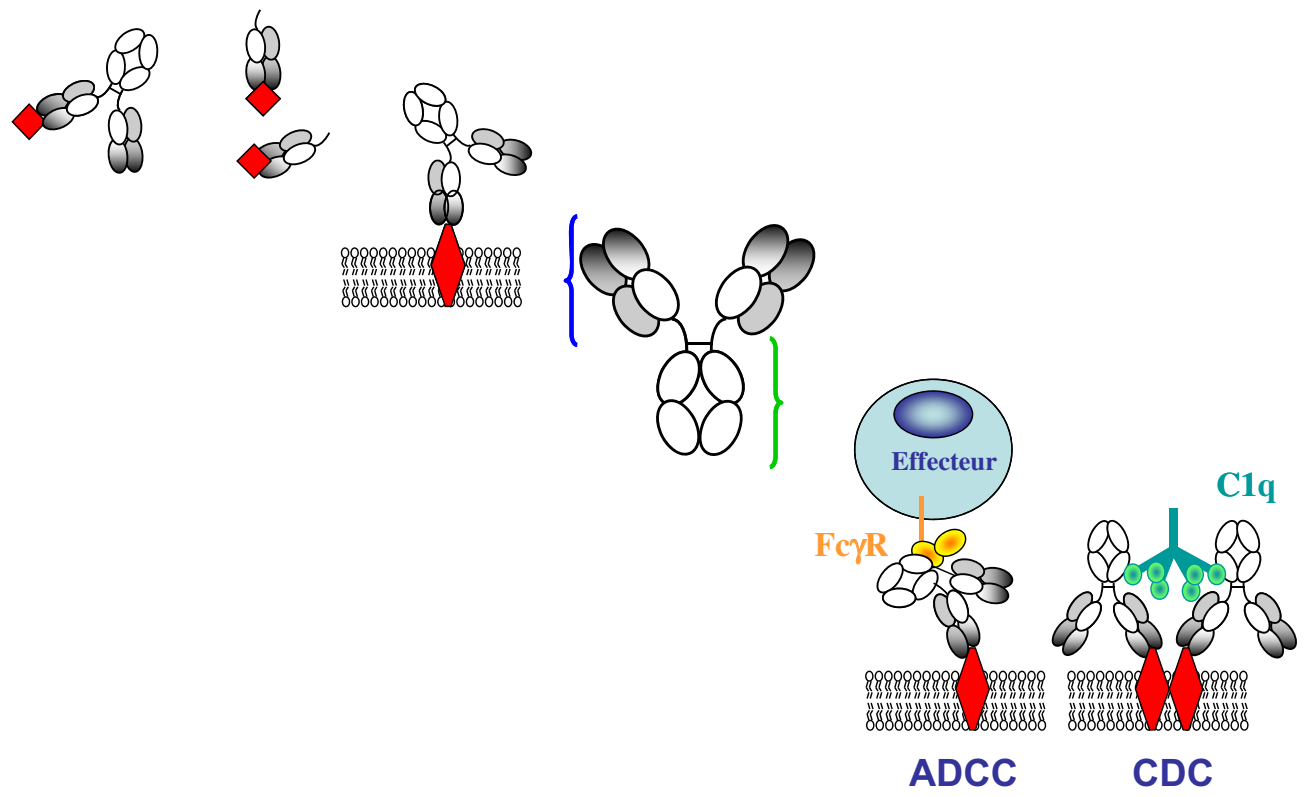

Fig. 2. Pour les AcMo neutralisants ou agonistes, c'est la portion Fab qui est déterminante et notamment sont affinité pour l'antigène-cible (représenté en rouge), qu'il soit circulant ou membranaire. Pour les anticorps cytolytiques, la portion Fc joue également un rôle important car elle est responsable du recrutement des effecteurs immunitaires : fraction C1q du complément (complement dependent cytotoxicity ou CDC) ou cellules effectrices porteuses du récepteur FcyRIIIA/CD16 (antibody dependent cellular cytotoxicity ou ADCC).

AcMo totalement humain, l'adalimumab, a été commercialisé en 2002 et il existait 7 AcMo de ce type en 2011. Une grande majorité des AcMo sur le marché a des indications en oncologie, oncohématologie et dans les pathologies inflammatoires. Cependant, les champs thérapeutiques des AcMo sont de plus en plus larges, concernant des domaines tels que l'ophtalmologie, l'auto-immunité (lupus), le système cardiovasculaire, les infections, la neurologie (sclérose en plaque, maladie d'Alzheimer) et les affections métaboliques (ostéoporose, hypercholestérolémie, diabète).

Pour un industriel, l'intérêt médico-économique de développer un AcMo repose sur les points suivants :

- l'efficacité thérapeutique est parfois spectaculaire. Une action sur la physiopathologie de la maladie est possible ;

- la connaissance des cibles potentielles est en essor. Le nombre des antigènes pouvant être ciblés par des AcMo est potentiellement infini, la seule condition étant que l'antigène soit accessible à l'anticorps, c'est-à-dire qu'il soit extracellulaire ;

- l'attrition des AcMo lors du développement clinique est plus faible que celle des médicaments «classiques » (petites molécules), notamment parce qu'ils sont responsables de moins d'interactions médicamenteuses et de moins d'effets indésirables à court terme ;

- les AcMo ayant été développés essentiellement dans des pathologies ou indications rares, où le besoin est important, ils ont été considérés majoritairement comme des progrès par la commis- sion de la transparence (obtention de niveaux d'amélioration du service médical rendu [ASMR] $>\mathrm{V}$ ).

Cependant, ceci est contre balancé par les éléments suivants :

- l'immunogénicité des AcMo peut entrainer la synthèse par le patient d'anticorps anti-AcMo, responsables d'un échappement thérapeutique et/ou d'effets indésirables ;

- des effets indésirables à long terme d'origine immunologique, parfois dramatiques (leucoencéphalopathie multifocale progressive [LEMP]), ont été observés ;

- bien que son antigène-cible soit parfaitement défini, la connaissance du mécanisme d'action de l'AcMo chez l'homme reste souvent imparfaite. La spécificité de la cible n'implique pas nécessairement une thérapeutique « ciblée »;

- pour un AcMo actif dans plusieurs pathologies, le développement doit être spécifique de la pathologie étudiée ;

- certains mécanismes effecteurs peuvent dépendre du potentiel de réponse du système immunitaire du patient, qui peut être altéré par la pathologie et les traitements associés ( $c f$ infra).

\subsection{Recommandations des agences réglementaires}

Un certain nombre de recommandations (guidelines) concernant le développement des AcMo ont été rédigées par la Food and Drug Administration (FDA), l'Agence européenne du médicament 
(EMA) et l'Agence française de sécurité sanitaire des produits de santé (Afssaps, devenue en 2012 l'Agence nationale de sécurité du médicament et des produits de santé [ANSM]). Des recommandations concernant la qualité des biomédicaments, dont celle des AcMo, ont été publiées en 2008 par l'EMA. ${ }^{[12]}$ Des recommandations existent dans le domaine des études de sécurité préclinique des biomédicaments. Il existe également des recommandations sur l'étude de l'immunogénicité des biomédicaments ${ }^{[13]}$ et, plus spécifiquement, des AcMo. ${ }^{[14]}$ Suite à l' affaire Tegenero (défaillances multiviscérales aiguës survenues chez des volontaires sains ayant reçu un AcMo anti-CD28 agoniste), ${ }^{[15]}$ l'ex-Afssaps $^{[16]}$ puis l'EMA ${ }^{[17]}$ ont publié des recommandations pour atténuer les risques d'effets indésirables lors de la 1ère administration à l'homme d'un médicament innovant. Des recommandations récentes concernent le développement des biosimilaires, incluant les AcMo. ${ }^{[18]}$ En revanche, il n'existe pas de recommandation sur le développement clinique des AcMo.

\subsection{Limites des modèles animaux}

Les modèles animaux sont d'utilisation délicate lors du développement des AcMo en raison des différences inter-espèces des « partenaires » de l'AcMo que sont l'antigène-cible et les effecteurs immunitaires (dont les récepteurs Fc $\gamma \mathrm{R}$ ). ${ }^{[19]}$ Les AcMo ne reconnaissent habituellement pas d'antigène-cible chez la souris. Ces modèles nécessitent donc d'utiliser un AcMo de subtitution (surrogate) se fixant aussi sur les homologues du récepteur chez l'animal ou d'utiliser des animaux transgéniques exprimant l'antigène-cible humain. Une dernière option est d'utiliser un AcMo reconnaissant un antigène de substitution, différent de celui ciblé chez l'homme mais entraînant des effets similaires. Par ailleurs, l'anticorps testé étant chimérique voire humanisé ou humain, il est rapidement immunogène pour l'animal servant de modèle. Compte tenu de ces limitations, les modèles cellulaires humains jouent un rôle très important dans le développement préclinique.

\subsection{Anticorps cytolytiques (cancérologie)}

Le mode d'action des AcMo chez l'homme reste encore mal connu. Cependant, une ADCC a été montrée pour certains anticorps, notamment grâce aux études pharmacogénétiques. Le nombre et l'efficacité des effecteurs immunitaires du patient vont donc influencer sa réponse clinique. Ceci peut avoir des conséquences, notamment en cancérologie, où les AcMo sont fréquemment testés en $2^{\mathrm{e}}$ ou $3^{\mathrm{e}}$ ligne, surtout dans les phases précoces du développement clinique. Les patients sont à un stade avancé de leur maladie et ont déjà été traités par une chimiothérapie cytotoxique qui peut avoir affecté leur immunité cellulaire. Il faudrait donc pouvoir tester l'AcMo chez des patients qui ne sont pas à un stade trop avancé de leur maladie et il est recommandé d'explorer de façon plus systématique les effecteurs cellulaires (notamment les cellules NK) en réalisant une numération des sous-populations lymphocytaires mais aussi en étudiant leurs fonctions ex vivo. Il est actuellement difficile de prédire les capacités de réponse à partir d'évaluations immunologiques, faute de tests standardisés. Cependant, cette documentation de l'état immunitaire des patients inclus permettra de comprendre les sources de variabilité interindividuelle de réponse lors des phases précoces, voire de ne pas sous-estimer l'efficacité d'un AcMo qui aurait été testé chez des patients ayant des effecteurs immunitaires peu actifs en raison de leur pathologie, des traitements antérieurs ou associés, ou d'un génotype particulier. Il serait également intéressant de pouvoir mesurer l'infiltration tumorale par les cellules NK car elle serait un bon biomarqueur de la réponse à l'AcMo. L'étude du génotype du récepteur FcyRIIIA chez les patients inclus est également justifiée.

\subsection{Immunogénicité}

Le risque d'immunisation contre l'AcMo semble inexistant dans le cancer. En revanche, dans les maladies autoimmunes, l'humanisation n'a pas fait disparaître les problèmes d'immunogénicité puisque $28 \%$ des patients se sont immunisés contre l'adalimumab, un AcMo totalement humain, après 3 ans de traitement, dont les $2 / 3$ durant les 28 premières semaines. ${ }^{[3]}$ Il est nécessaire d'explorer les conséquences cliniques de cette immunisation. Il s'agit principalement d'une chute des concentrations d'AcMo associée à un échappement thérapeutique mais également d'effets indésirables sévères liés à cette immunisation. ${ }^{[3]}$ Des tests in vitro prédisant l'immunogénicité des nouveaux biomédicaments sont proposés mais leur performance est encore mal connue.

\subsection{Phases précoces (I et II)}

Dès les premières administrations à l'homme, la qualité des lots cliniques est capitale, notamment parce que les agrégats sont un facteur important d'immunogénicité. L'étude du mécanisme d'action des AcMo chez l'homme in vivo est difficile et il existe beaucoup de pièges. Le risque de réactions immunologiques suivant les premières doses peut correspondre :

- au syndrome de relargage des cytokines (fréquent) ;

- à des réactions anaphylactiques (médiées par des IgE) [rares] ;

- à des réactions croisées, liées à la présence de l'antigène-cible dans les tissus sains (exemple : AcMo anti-EGFR et rash cutanés).

Les guidelines recommandent un calcul de la $1^{\mathrm{re}}$ dose reposant sur la minimum anticipated biological effect level (MABEL) plutôt que sur la no observed adverse effect level (NOAEL). ${ }^{[17]}$

Cependant :

- c'est une approche très conservatrice, qui prolonge de façon importante la durée des essais de phase précoce. Elle entraîne par 


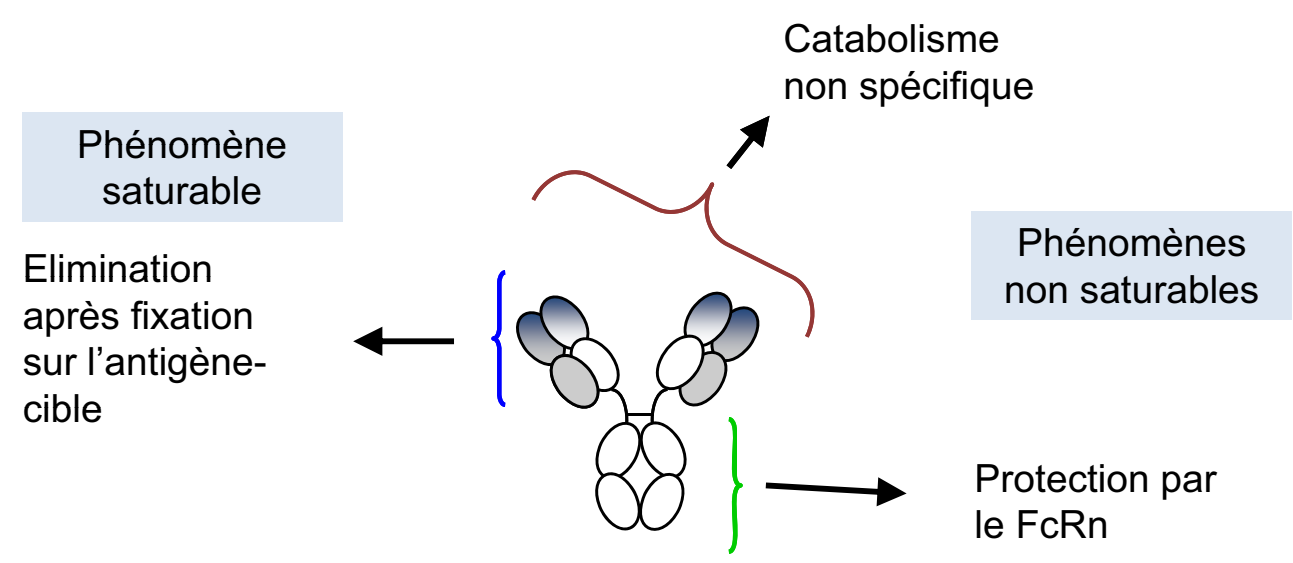

Fig. 3. Les AcMo sont éliminés par catabolisme non spécifique et en partie protégés par le récepteur Fc néonatal ou FcRn. Ces deux phénoèmes ne sont pas saturables. Ils sont également éliminés après fixation sur l'antigène-cible. Ce dernier étant, par définition, en quantité limitée, ce mode d'élimination des AcMo est saturable.

ailleurs un risque de perte de chance pour les patients recevant de faibles doses ;

- elle semble surtout nécessaire pour les AcMo ayant un nouveau mécanisme d'action et ayant une cible membranaire, pour lesquels des effets indésirables aigus d'origine immunologique ne peuvent être exclus ;

- elle nécessite une expertise immunologique pour tenter d'extrapoler à l'homme les résultats observés dans les modèles précliniques animaux ou cellulaires ;

- par précaution, lors de la toute première administration (et parfois à chaque palier de dose), il peut être recommandé de n'exposer qu'un tout petit nombre de sujets/patients à la fois ( 1 ou 2 sujets « sentinelles ») avant d'exposer la cohorte entière.

La question de la population recrutée pour la $1^{\text {re }}$ administration se pose également : faut-il étudier des sujets sains ou des patients ? Le choix est fondé sur des considérations éthiques (rapport bénéfice/risque) et scientifiques, notamment la présence de la cible et les effets biologiques et cliniques attendus, mais il peut modifier profondément les résultats. D'autres questions restent ouvertes :

- faut-il choisir des doses adaptées au poids ou des doses fixes ? Cette dernière approche semble plus pertinente ;

- faut-il faire les premières administrations par voie intraveineuse (pour pouvoir interrompre la perfusion en cas de réaction immédiate) ou directement par voie sous-cutanée si cette dernière est la voie choisie pour le développement?

Lors de la phase II, le choix raisonné de la dose est difficile pour deux raisons :

- il faut disposer de bons biomarqueurs, notamment d'un « immuno-monitoring », mais il y a souvent une cascade d'évènements entre les biomarqueurs mesurés et l'effet thérapeutique. Cette « déconnection », au minimum temporelle, de l'effet thérapeutique et des biomarqueurs rend difficile l'interprétation des études pharmacocinétiques-pharmacodynamiques (PK-PD);

- la relation concentration - toxicité est mal connue. En effet, la toxicité est essentiellement dépendante de la cible, contrairement aux médicaments «classiques » : il s'agit d'une exagération de l'activité pharmacodynamique ou d'une «accumulation» à long terme de l'effet, par exemple sous la forme d'une immunosuppression.

L'analyse des AcMo actuellement sur le marché montre que le choix des doses semble avoir été parfois empirique. Cela reflète la difficulté du développement clinique des AcMo, notamment celle des phases précoces, qui sont probablement insuffisamment codifiées.

\subsection{Pharmacocinétique}

Après administration sous-cutanée ou intra-musculaire, l'absorption des AcMo est très lente, avec un temps de pic autour d'une semaine. Les mécanismes d'élimination des AcMo sont très différents des médicaments « classiques ». D'une part, ils subissent un catabolisme non spécifique, les IgG étant dégradées comme les autres protéines circulantes par les cellules endothéliales vasculaires, ce phénomène n'étant pas saturable. D'autre part, les AcMo sont éliminés après fixation sur leur cible, par internalisation lorsque la cible est un récepteur membranaire ou par formation de complexes immuns lorsque la cible est circulante. ${ }^{[20]} \mathrm{La}$ quantité de cible étant, par définition, limitée, ce mode d'élimination des AcMo est saturable. Le troisième mécanisme intervenant dans l'élimination des AcMo est leur protection contre la dégradation grâce à un récepteur particulier, le récepteur Fc néonatal ou FcRn. ${ }^{[21]}$ Cette protection explique leur longue demi-vie d'environ 3 semaines (figure 3). Lorsque les protéines circulantes sont captées de façon passive par les cellules endothéliales vasculaires, les 


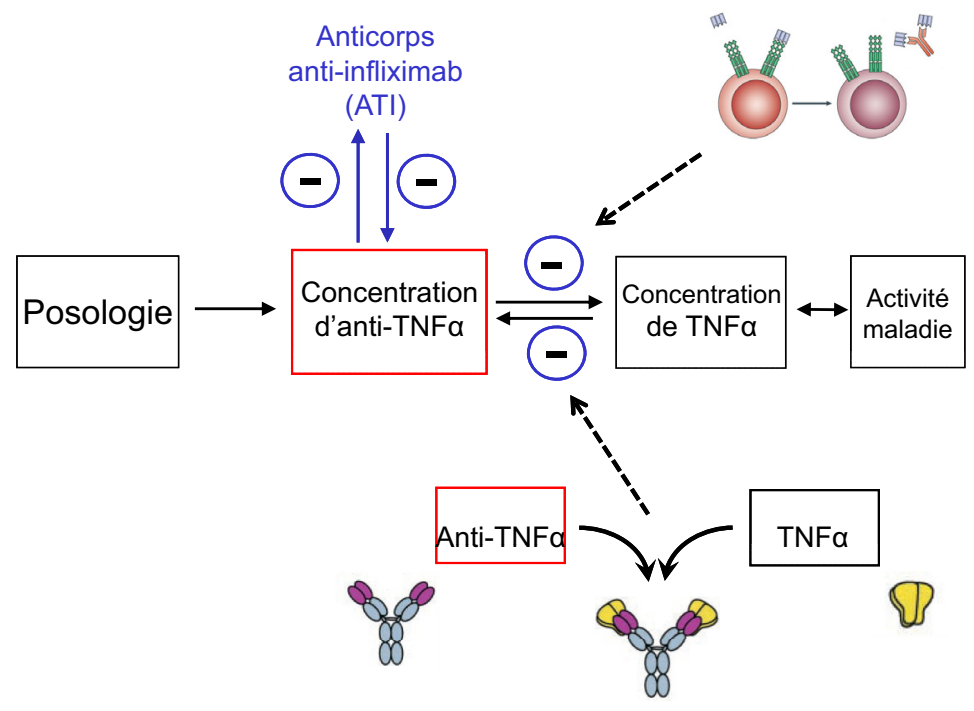

Fig. 4. L'élimination des AcMo après fixation sur leur antigène-cible implique que la quantité d'antigène-cible va influencer la pharmacocinétique, comme dans l'exemple des AcMo anti-TNF $\alpha$. Le risque d'immunisation est d'autant plus important que les concentrations d'AcMo sont faibles. Lorsqu'une immunisation s'est produite, les anticorps anti-AcMo vont diminuer les concentrations de l'AcMo. II existe donc des inter-relations entre AcMo, antigène-cible et anticorps induits. En particulier, si l'activité de la maladie est importante, avec concentrations élevées d'antigène-cible, les concentrations d'AcMo seront diminuées et le risque d'immunisation et donc d'échappement thérapeutique sera augmenté.

endosomes s'acidifient progressivement et les protéines sont dégradées dans des lysosomes. Le FcRn, présent dans les vésicules d'endocytose, fixe les anticorps au niveau de leur portion Fc et les détourne de cette voie de dégradation pour les rediriger vers la membrane apicale de la cellule. Aux concentrations thérapeutiques des AcMo, ce phénomène n'est pas saturable. En terme de modélisation pharmacocinétique, l'élimination des AcMo est donc habituellement non linéaire et doit être décrite à la fois par des phénomènes non saturables et par des phénomènes saturables. Le FcRn, qui est présent dans de nombreux tissus, est également responsable de la transcytose des anticorps et donc de leur distribution tissulaire. Les anticorps ne sont donc pas confinés dans la circulation systémique. Le FcRn est responsable notamment du passage transplacentaire des anticorps maternels (anticorps naturels ou AcMo) en fin de grossesse, de l'expulsion des anticorps du système nerveux central (ce qui explique le faible passage des AcMo injectés par voie intra-veineuse). ${ }^{[20,21]}$

Certaines sources de variabilité interindividuelle de la pharmacocinétique des AcMo sont différentes de celles des médicaments «classiques ». Puisque la fixation sur l'antigène-cible entraîne l'élimination de l'anticorps, la « masse antigénique » va influencer la pharmacocinétique. ${ }^{[22]}$ En effet, la quantité d'antigène-cible est variable selon les patients, que ce soit dans les maladies tumorales ou dans les maladies inflammatoires. L'activité de la maladie va donc influencer la clairance des AcMo (figure 4). Cette relation à double sens doit idéalement être décrite par un modèle de type « élimination liée à la cible » (target-mediated drug disposition ou
TMDD), qui permet de décrire de façon conjointe la pharmacocinétique et la relation PK-PD. ${ }^{[23]}$

Comme nous l'avons vu plus haut, l'immunisation des patients va être responsable d'une diminution des concentrations de l'AcMo. Cette immunisation est elle-même dépendante des concentrations de l'anticorps thérapeutique. Il a en effet été montré que le risque de développer des anticorps anti-infliximab est d'autant plus élevé que les concentrations d'infliximab sont faibles. ${ }^{[24,25]}$ L'activité de la maladie inflammatoire pourrait donc être indirectement responsable de l'apparition d'anticorps induits et donc des échappements thérapeutiques secondaires (figure 4). Par ailleurs, des anticorps anti-AcMo peuvent être présents avant la première administration du médicament, comme cela a été montré pour les $\operatorname{IgE}$ anti- $\alpha$ GAL dirigés contre le cetuximab ${ }^{[26]}$ et lorsque la portion Fc de l'AcMo a été modifiée. [27]

\section{4. Évaluation par les agences et suivi de tolérance à long terme}

\section{1. Évaluation par la commission d'AMM de l'ex-Afssaps}

L'AMM des AcMo, en tant que médicaments issus de procédés biotechnologiques, sont octroyées dans le cadre d'une procédure centralisée européenne après évaluation par l'EMA, deux 
pays étant rapporteurs et les autres destinataires. Dans la mesure où l'expertise spécifique à ces produits biotechnologiques doit être associée à celle de la pathologie ciblée, il n'apparait pas nécessaire de mettre en place un groupe d'évaluation spécialisé auprès de la commission d'AMM qui serait exclusivement en charge de l'évaluation des AcMo.

\section{2. Évaluation par la commission de transparence de la Haute autorité de santé}

Les AcMo ont de multiples indications. Depuis 1999, la commission de transparence de la Haute autorité de santé (HAS) a analysé en vue de leur remboursement 22 spécialités dans 45 indications: 12 avec 1 indication, 5 avec 2 indications, 1 avec 4 indications et 4 avec 5 indications (une indication pouvant obtenir plusieurs niveaux d'ASMR, par exemple selon la ligne de traitement). Le service médical rendu (SMR) a été considéré comme important 42 fois et modéré ou faible 4 fois. L'ASMR a été considérée comme majeure (de niveau I) à modérée (de niveau III) 23 fois et mineure (niveau IV) 11 fois. Dans 11 cas, un AcMo n'a pas été considéré comme un progrès par rapport à l'existant (ASMR $\mathrm{V})$ et 1 fois l'ASMR n'a pas pu être appréciée. L'évaluation des AcMo n'est pas différente de celle des autres médicaments (la même grille de lecture est utilisée), mais les situations de traitement (gravité et/ou rareté de la maladie) ont, jusqu'à présent, été particulières.

\section{3. Études de tolérance à long terme}

Les études de tolérance ne constituent qu'une partie des études post-AMM demandées par la commission de transparence de la HAS. Sur un total de 166 études demandées, 32 études ont concerné 15 spécialités d'AcMo : 15 études sur les conditions de prescription/d'utilisation, 9 études sur le bénéfice en morbi-mortalité pour les patients, 2 études d'impact sur le système de soins et 6 études de la tolérance. Dans le cas des AcMo, les études de tolérance à long terme semblent justifiées par :

- la gravité ou la rareté de la maladie ;

- le caractère nouveau de l'antigène-cible, les conséquences cliniques de son interaction avec l'AcMo étant mal connues ;

- l'évaluation incomplète des modalités de prescription (dose et durée de traitement) ;

- le découplage des types de toxicité observés à court terme de ceux observés à long terme ainsi que l'absence de données sur la toxicité à long terme au moment de l'évaluation pour la mise sur le marché et la première inscription au remboursement.

La table ronde s'est interrogée sur la possibilité de mieux orienter les plans de gestion des risques (PGR) post-AMM demandés par l'EMA (ou spécifiquement par l'Afssaps) pour les AcMo, certains effets indésirables étant attendus compte tenu de leur mode d'action relativement spécifique (cible de l'AcMo parfaitement connue) et de la connaissance du format de l'AcMo (cytolyse attendue s'il s'agit d'une IgG1 par exemple). Cependant, il persiste des effets inattendus et certains effets attendus ne sont pas observés. Il est clair que l'accompagnement du développement des AcMo par une expertise immunologique est essentiel afin d'aider à en prévoir l'ensemble des effets mais, qu'en retour, l'utilisation des AcMo a fait progresser les connaissances en immunologie. La prédiction des effets indésirables ne peut donc qu'être encore imparfaite et la prudence reste nécessaire.

\subsection{Observatoires des médicaments, des dispositifs médi- caux et de l'innovation thérapeutique}

Les Observatoires des médicaments, des dispositifs médicaux et de l'innovation thérapeutique (OMEDITs) permettent d'analyser les conditions de prescription des AcMo dans la « vie réelle », de réaliser des études coût/efficacité et de répondre aux demandes faites par la commission de transparence dans le cadre du suivi post-AMM des AcMo. L'observatoire dédié au cancer des régions Bretagne et Pays de la Loire est rattaché aux deux OMEDITs de ces régions. Cet observatoire a par exemple réalisé une analyse des coûts engendrés pour traiter 730 patients pour cancer colorectal métastatique dans 38 établissements pour une ligne de traitement sur un an d'observation : la médiane de coût par malade était de $7500 €$ pour la chimiothérapie seule contre $24500 €$ pour la chimiothérapie associée à un AcMo. Le poste « médicament » était plus élevé pour les AcMo tandis qu'il était moindre pour les toxicités. ${ }^{[28]}$ Une autre étude de cet observatoire a porté sur les conditions réelles d'utilisation d'une association bevacizumab + chimiothérapie dans le cancer colorectal métastatique chez 2050 patients, et a observé, par rapport aux données de la littérature, ${ }^{[29]}$ un âge moyen, un risque de toxicité et un risque de décès plus élevés. ${ }^{[30]}$ Les études post-AMM sont indispensables car elles apportent des informations sur la population effectivement traitée et sur le rapport bénéfice/risque dans cette population.

\subsection{Place des sociétés savantes}

La HAS rédige des recommandations sur la prescription des médicaments. Son objectif est d'éclairer la décision publique et les textes peuvent donc être en décalage par rapport à ceux rédigés par les sociétés savantes, qui renseignent plus sur l'état de l'art pour le prescripteur. Il est important de disposer d'avis les plus indépendants possibles de conflits d'intérêt. Les thèmes des recommandations sont choisis par le collège de la HAS selon les priorités de santé publique et sur demandes du ministre, des sociétés savantes, de l'assurance maladie, des associations de patients ou d'autres organisations professionnelles. La rédaction est coordonnée par un comité technique qui s'appuie sur des groupes de travail multidisciplinaires et des relecteurs externes. La version finale est validée par le collège de la HAS avant diffusion. Cette procédure peut donc 
prendre plus de temps (environ 18 mois) que la rédaction des recommandations ayant d'autres sources.

Les suivis post-AMM peuvent également être réalisés dans le cadre de registres, qui sont des suivis de cohortes de patients traités par AcMo, mis en place par des sociétés savantes, souvent en partenariat avec les agences et/ou les industriels, ces derniers n'étant cependant pas impliqués dans l'élaboration et la conduite du suivi. L'exemple de la rhumatologie a été discuté lors de la table ronde, 20 registres européens ayant été identifiés pour cette discipline dont 4 en France : «RATIO », programme de pharmacovigilance approfondi pour le recueil des infections opportunistes et des lymphomes chez les patients traités par anti-TNF, «AIR », suivi de patients traités par rituximab pour maladies auto-immunes, «ORA », suivi de patients traités par abatacept pour polyarthrite rhumatoïde, et enfin «REGATE », suivi des patients traités par tocilizumab pour polyarthrite rhumatoïde. ${ }^{[31]}$ Bien que ces registres permettent de recueillir beaucoup d'informations, une meilleure coordination des différents acteurs (sociétés savantes, industrie pharmaceutique, agences) serait souhaitable.

\section{Conclusion}

Les AcMo, qui peuvent agir sur un nombre potentiellement infini de cibles et de fonctions, sont des médicaments innovants : la nouveauté de leurs modes d'action constitue un défi pour la pharmacologie clinique. La table ronde n'a pas identifié une caractéristique qui justifierait que la méthodologie de leur développement clinique et de leur évaluation soit spécifique. Cependant, l'analyse a permis de mettre en évidence un ensemble de particularités des AcMo par rapport aux médicaments « classiques » :

- les phases précoces du développement clinique posent des problèmes spécifiques, notamment quant au choix de la $1^{\text {re }}$ dose à tester et des sujets à inclure ;

- la pharmacocinétique des AcMo est très différente de celle des médicaments « classiques »;

- la toxicité est essentiellement dépendante de la cible, avec exagération de l'activité pharmacodynamique et/ou «accumulation » des effets immunosuppresseurs pour certains d'entre eux ;

- la relation PK-PD est difficile à étudier car l'effet thérapeutique peut être lié à une cascade d'évènements et déconnecté des biomarqueurs accessibles ;

- l'effet thérapeutique des AcMo cytolytiques sollicite le système immunitaire du patient, qui est variable pour des raisons génétiques, physiologiques et pathologiques.

Si l'évaluation des AcMo par les autorités de santé ne se différencie pas de celle des médicaments « classiques », la particularité des AcMo provient de la conjonction de plusieurs facteurs : les maladies traitées sont graves et/ou rares, il s'agit souvent d'un nouvel antigène-cible, les modalités de prescription (dose et durée de traitement) sont mal évaluées durant le développement clinique et il existe un découplage de la toxicité à court terme et de la toxicité à long terme, cette dernière restant mal décrite lors de la mise sur le marché.

\section{Participants.}

Martine Bagot (Université Paris Diderot Sorbonne, AP-HP Hôpital Saint-Louis, Inserm U976), Isabelle Boucot (GSK), Anne d'Andon (Haute Autorité de Santé), Françoise Grudé (Observatoire dédié au Cancer Bretagne Pays de la Loire, ICO Paul Papin Angers), Thierry Lecomte (Université François Rabelais Tours, CHRU de Tours, CNRS UMR 7292), Soyi Liu-Leage (Lilly), Daniel Olive (Aix-Marseille Université, Institut Paoli Calmettes, Inserm), Pascal Piedbois (Astra Zeneca), Martine Pigeon (CNAMTS), Jean-Louis Pinquier (Sanofi R\&D), Jean-François Prost (LFB), Gilles Thibault (Université François Rabelais Tours, CHRU de Tours, CNRS UMR 7292), Christian Vitzling (Novartis).

Conflits d'intérêts. Gilles Paintaud dirige une équipe de recherche recevant des financements du Laboratoire français du fractionnement et des biotechnologies (LFB) ainsi que des Laboratoires Pierre Fabre, Novartis, Roche Pharma, Pfizer, Janssen et Chugai. Marine Diviné est salariée d'Amgen France. Philippe Lechat n'a pas de conflit d'intérêt à déclarer.

Abréviations. AcMo : anticorps monoclonaux ; ADCC : antibody dependent cellular cytotoxicity; Afssaps : Agence française de sécurité sanitaire des produits de santé ; of ${ }^{1}$ AMM : autorisation de mise sur le marché ; ANSM : Agence nationale du médicament et des produits de santé ; anti-RANK-ligand : anti-receptor activator of nuclear factor-kappaB ligand; anti-TNF $\alpha$ : anti-tumor necrosis factor $\alpha$; anti-VGEF : anti-vascular endothelial growth factor; ASMR : amélioration du service médical rendu ; CDC: complement dependent toxicity ; CDR : complementary determining region; EMA : Agence européenne du médicament ; FDA : Food and Drug Administration ; HAS : Haute autorité de santé; HER : récepteurs aux facteurs épidermiques ; IgG : immunoglobulines $G$; LEMP : leucoencéphalopathie multifocale progressive ; MABEL : minimum anticipated biological effect level; NK : natural killer ; NOAEL : no observed adverse effect level ; OMEDITs : Observatoires des médicaments, des dispositifs médicaux et de l'innovation thérapeutique ; of ${ }^{2}$ PGR : plans de gestion des risques ; PK-PD : pharmacocinétique-pharmacodynamique ; SMR : service médical rendu ; of ${ }^{3}$ et ${ }^{4}$ TMDD : target-mediated drug disposition.

1. AIR : suivi de patients traités par rituximab pour maladies autoimminences ;

2. ORA : suivi de patients traités par abatacept pour polyarthrite rhumatoïde ;

3. RATIO : programme de pharmacovigilance approfondi pour le recueil des infections opportunistes et des lymphomes chez les patients traités par anti-TNF ;

4. REGATE : suivi des patients traités par tocilizumab par polyarthrite rhumatoide. 


\section{Références}

1. Paintaud G, Tonelli D, Postaire E, et al. Biothérapies : des médicaments comme les autres ? Therapie 2007; 62: 229-34

2. Paintaud G, Lejarre F, Ternant D, et al. Les anticorps monoclonaux : une avancée thérapeutique récente et majeure. Therapie 2009; 64: 1-7

3. Bartelds GM, Krieckaert CL, Nurmohamed MT, et al. Development of antidrug antibodies against adalimumab and association with disease activity and treatment failure during long-term follow-up. JAMA 2011; 305: 1460-8

4. Mitoma $\mathrm{H}$, Horiuchi $\mathrm{T}$, Tsukamoto $\mathrm{H}$, et al. Mechanisms for cytotoxic effects of anti-tumor necrosis factor agents on transmembrane tumor necrosis factor alpha-expressing cells: comparison among infliximab, etanercept, and adalimumab. Arthritis Rheum 2008; 58: 1248-57

5. Congy-Jolivet N, Probst A, Watier H, et al. Recombinant therapeutic monoclonal antibodies: mechanisms of action in relation to structural and functional duality. Crit Rev Oncol Hematol 2007; 64: 226-33

6. Lejeune $\mathrm{J}$, Thibault $\mathrm{G}$, Cartron $\mathrm{G}$, et al. Rôle des récepteurs à la portion Fc des IgG (Fc Rs) dans l'activité des anticorps thérapeutiques. Bull Cancer 2010; 97: 511-22

7. Cartron G, Dacheux L, Salles G, et al. Therapeutic activity of humanized anti-CD20 monoclonal antibody and polymorphism in IgG Fc receptor FcgammaRIIIa gene. Blood 2002; 99: 754-8

8. Musolino A, Naldi N, Bortesi B, et al. Immunoglobulin G fragment C receptor polymorphisms and clinical efficacy of trastuzumab-based therapy in patients with HER-2/neu-positive metastatic breast cancer. J Clin Oncol 2008; 26: 1789-96

9. Bibeau F, Lopez-Crapez E, Di Fiore F, et al. Impact of Fc $\gamma R$ IIa-Fc $\gamma R$ IIIa polymorphisms and KRAS mutations on the clinical outcome of patients with metastatic colorectal cancer treated with cetuximab plus irinotecan. $\mathbf{J}$ Clin Oncol 2009; 27: 1122-9

10. Béliard R, Waegemans T, Notelet D, et al. A human anti-D monoclonal antibody selected for enhanced Fc $\gamma \mathrm{RIII}$ engagement clears $\mathrm{RhD}^{+}$autologous red cells in human volunteers as efficiently as polyclonal anti-D antibodies. Br J Haematol 2008; 141: 109-19

11. De Romeuf C, Dutertre CA, Le Garff-Tavernier M, et al. Chronic lymphocytic leukaemia cells are efficiently killed by an anti-CD20 monoclonal antibody selected for improved engagement of Fc $\gamma$ RIIIA/CD16. Br J Haematol 2008; 140: 635-43

12. EMA. Guideline on development, production, characterization and specifications for monoclonal antibodies and related products. $2008 \mathrm{CHMP} / \mathrm{BWP} /$ 157653/2007. http://www.ema.europa.eu

13. EMA. Guideline on immunogenicity assessment of biotechnology-derived therapeutic proteins. 2007 EMEA/CHMP/BMWP/14327/2006. http:// www.ema.europa.eu

14. EMA. Guideline on immunogenicity assessment of monoclonal antibodies intended for in vivo clinical use. 2010 EMEA/CHMP/BMWP/86289/2010. http://www.ema.europa.eu

15. Suntharalingam G, Perry MR, Ward S, et al. Cytokine storm in a phase 1 trial of the anti-CD28 monoclonal antibody TGN1412. N Engl J Med 2006; 355 : 1018-28

16. Afssaps. Essais cliniques de première administration à l'Homme, en dose unique d'un médicament expérimental (nouvelle substance active) : choix de la première dose, de la progression de dose et protocole d'administration aux volontaires. 2006. http://ansm.sante.fr/S-informer/Presse-Communiques-Points-presse/Premiere-administration-d-un-medicament-experimental-chez-I-homme-I-Afssaps-diffuse-des-recommandations-sur-lesessais-cliniques-de-phase-I/(language)/fre-FR
17. EMA. Guideline on requirements for first-in-man clinical trials for potential high-risk medicinal products. 2007 CHMP/SWP/28367/2007. http:// www.ema.europa.eu

18. EMA. Guideline on similar biological medicinal products containing monoclonal antibodies. 2010 EMEA/CHMP/BMWP/403543/2010. http:// www.ema.europa.eu

19. Loisel S, Ohresser M, Pallardy M, et al. Relevance, advantages and limitations of animal models used in the development of monoclonal antibodies for cancer treatment. Crit Rev Oncol Hematol 2007; 62: 34-42

20. Paintaud G. Pharmacocinétique des anticorps monoclonaux. Med Sci (Paris) 2009; 25: 1057-62

21. Magdelaine-Beuzelin C, Ohresser M, Watier H. FcRn, un récepteur d'IgG aux multiples facettes. Med Sci (Paris) 2009; 25: 1053-6

22. Ternant D, Paintaud G. Pharmacokinetics and concentration-effect relationships of therapeutic monoclonal antibodies and fusion proteins. Expert Opin Biol Ther 2005; 5: S37-S47

23. Yan X, Mager DE, Krzyzanski W. Selection between Michaelis-Menten and target-mediated drug disposition pharmacokinetic models. J Pharmacokinet Pharmacodyn 2009; 37: 25-47

24. Bendtzen K, Geborek P, Svenson M, et al. Individualized monitoring of drug bioavailability and immunogenicity in rheumatoid arthritis patients treated with the tumor necrosis factor inhibitor infliximab. Arthritis Rheum 2006; 54: 3782-9

25. Ducourau E, Mulleman D, Paintaud G, et al. Antibodies toward infliximab are associated with low infliximab concentration at treatment initiation and poor infliximab maintenance in rheumatic diseases. Arthritis Res Ther 2011; 13: R105

26. Chung $\mathrm{CH}$, Mirakhur B, Chan E, et al. Cetuximab-induced anaphylaxis and IgE specific for galactose-alpha-1,3-galactose. N Engl J Med 2008; 358: 1109-17

27. Zheng Y, Scheerens H, Davis JC Jr, et al. Translational pharmacokinetics and pharmacodynamics of an FcRn-variant anti-CD4 monoclonal antibody from preclinical model to phase I study. Clin Pharmacol Ther 2011; 89: 283-90

28. Grudé F, Roué V, Beauchamp C, et al. Real cost linked to the introduction of targeted therapies (bevacizumab, cetuximab) and conventional chemotherapies in the treatment of metastatic colorectal cancer. Gastrointestinal Cancers Symposium, San Francisco 2009, A330

29. Hurwitz HI, Fehrenbacher L, Hainsworth JD, et al. Bevacizumab in combination with fluorouracil and leucovorin: an active regimen for first-line metastatic colorectal cancer. J Clin Oncol 2005; 23: 3502-8

30. Metges JP, Grudé F, Faroux R, et al. FOLFIRI bevacizumab in unresectable metastatic colorectal cancer in the true life, feasible in elderly patients: results of the cohort from OMIT Bretagne-Pays de Loire. Gastrointestinal Cancers Symposium, San Francisco, A398

31. Mariette X, Gottenberg JE, Ravaud P, et al. Registries in rheumatoid arthritis and autoimmune diseases: data from the French registries. Rheumatology $2011 ; 50: 222-9$

Correspondance et offprints: Gilles Paintaud, Laboratoire de Pharmacologie-Toxicologie et CNRS UMR 7292, CHRU de Tours, 2 boulevard Tonnellé, 37044 Tours Cedex 9, France.

E-mail : paintaud@med.univ-tours.fr 